DOI - 10.21707/gs.v10.n02a09

\title{
O CONTRIBUTO DO TURISMO PARA O DESENVOLVIMENTO DAS REGIÕES
}

\author{
Catarina A. Martins ${ }^{1} \&$ Antónia M. Morais ${ }^{2}$
}

\begin{abstract}
${ }^{1}$ Licenciatura em Gestão e Planeamento em Turismo pela Universidade de Aveiro; 2002. Mestrado em Contabilidade e Administração pela Universidade do Minho; Doutoranda em Turismo na Universidade de Aveiro desde 2010. Professora Adjunta na Escola Superior de Comunicação Administração e Turismo do Instituto Politécnico de Bragança.

${ }^{2}$ Licenciada em Ciências da Comunicação, pela Universidade Fernando Pessoa; 1997. Pós-graduação em Assuntos Culturais no Âmbito das Autarquias, pela Faculdade de Letras da Universidade de Coimbra; 2008. Pós-graduação em Turismo, Recursos Locais, Animação e Desenvolvimento, pela Universidade de Trás-os-Montes e Alto Douro. Técnica Superior na Câmara Municipal de Macedo de Cavaleiros.
\end{abstract}

Recebido em 20 de fevereiro de 2016. Aceito em 10 de setembro de 2016. Publicado em 09 de dezembro de 2016.

\begin{abstract}
Resumo - O turismo é uma atividade económica que cada vez mais surge como aposta essencial para o desenvolvimento das regiões. O presente trabalho resulta de uma extensa revisão da literatura que pretende essencialmente identificar quais os fatores que estão na base do desenvolvimento de uma região no que ao turismo diz respeito, designadamente, a atração de investimento externo para a região, a organização em rede e o estabelecimento de parcerias, a criação de produtos-território assentes em clusters para o turismo, a governância das redes e das instituições envolvidas no planeamento local e a importância dos critérios económicos. Discute-se ainda a dicotomia desenvolvimento versus crescimento e apresenta-se uma reflexão teórica em torno da importância do turismo sustentável no desenvolvimento das regiões.
\end{abstract}

Palavras-chave: Turismo, Desenvolvimento Sustentável, Regiões, Clusters

\section{THE CONTRIBUTION OF TOURISM TO THE REGIONS DEVELOPMENT}

ABstract - Tourism is an economic activity that increasingly appears to bet essential to the development of the regions. The present work is the result of an extensive literature review that aims to essentially identify what factors underlie the development of a region in what concerns the tourism industry, namely, the attraction of foreign investment to the region, the network organizations and the establishment of partnerships, the creation of products-territory based on clusters for tourism, the governance of networks and institutions involved in local planning and the importance of the economic criteria. The paper also discusses the dichotomy between development and growth and presents a theoretical reflection around the importance of sustainable tourism in the development of the regions.

Key words: Tourism, Sustainable Development, Regions, Clusters

\section{LA CONTRIBUCIÓN DEL TURISMO AL DESARROLLO DE LAS REGIONES}

REsumeN - El turismo es una actividad económica que surge cada vez más como apuesta esencial para el desarrollo de las regiones. El presente trabajo resulta de una extensa revisión de la literatura que pretende esencialmente identificar cuáles son los factores que están en la base del desarrollo de una región en lo que al turismo dice respeto, como son, la atracción de inversión extranjera para la región, la organización en red y el establecimiento de asociaciones, la creación de productos-territorio basados en clusters para el turismo, la governância de las redes y de las instituciones envueltas en la planificación local y la importancia de los criterios económicos. Se discute todavía la dicotomía desarrollo versus crecimiento y se presenta una reflexión teorética alrededor de la importancia del turismo sostenible en el desarrollo de las regiones.

Palavras clave: Turismo, Desarkollo Sostenible, Regiones, Clusters

\section{INTRODUÇÃO}

O turismo é uma atividade económica extremamente importante, podendo desempenhar um papel decisivo em termos de desenvolvimento de determinadas regiões, onde, por vezes, existem poucas alternativas para alcançar esse objetivo.

O turismo é uma das atividades em franco crescimento a nível global, surgindo como uma alternativa de desenvolvimento e crescimento das regiões. O turismo, graças à sua importância económica e sociocultural, é um importante pilar para a economia e desenvolvimento das regiões, pois é motor de crescimento de um conjunto de atividades relevantes e, por isso, os governos regionais e locais interessados em promover o desenvolvimento veem no turismo um forte aliado na procura desse desenvolvimento. O desenvolvimento deve ser visto como um conjunto de ações, mecanismos, estratégias e políticas endógenas, desencadeadas por atores locais em interação com as demais escalas de poder e gestão, reforçando e constituindo articulações por meio de novos usos políticos e económicos do espaço regional.
Neste artigo, proceder-se-á, num primeiro momento, a um breve enquadramento e conceptualização no que respeita à dicotomia no processo de desenvolvimento e crescimento das regiões, designadamente, no que respeita ao desenvolvimento sustentado. Posteriormente, identificam-se cinco fatores que poderão estar na base do desenvolvimento de uma região no que ao turismo diz respeito, designadamente, a atração de investimento externo para a região, a organização em rede e o estabelecimento de parcerias, a criação de produtos-território assentes em clusters para o turismo, a governança das redes e das instituições envolvidas no planeamento local, e por último, a importância dos critérios económicos.

\section{A Dicotomia No PROCESSO DE DESENVOLVIMENTO/ CRESCIMENTO}

O contributo de um qualquer sector para o desenvolvimento de um país ou de uma região deve ser visto à luz daquelas que são as premissas inerentes ao crescimento económico e ao desenvolvimento económico. Estes dois conceitos estão intimamente relacionados e um não existe 
sem o outro. Isto é, não teremos desenvolvimento sem crescimento económico assim como o crescimento económico não será relevante se não permitir a seguir o desenvolvimento económico.

O crescimento económico de um país é definido como sendo um aumento de longo prazo na capacidade de fornecer, de modo crescente, bens económicos diversos à população, sendo esta capacidade crescente baseada em tecnologia avançada e nos ajustamentos institucionais e ideológicos necessários (Kuznets, 1973) Para o autor as três componentes da definição são essenciais. O fornecimento em crescendo de bens é o resultado do crescimento económico, os avanços na tecnologia são a condição permissiva desse crescimento e os ajustamentos ideológicos e institucionais são necessários para permitir que o uso da tecnologia seja estimulado e empregue amplamente e de forma eficiente.

De acordo com a Enciclopédia Moderna Larousse, contrariamente ao crescimento, que implica o aumento sustentado e duradouro das principais grandezas que caracterizam um estado económico (produção, investimentos, rendimentos, consumo, etc.) a noção de desenvolvimento centra-se nos aspetos qualitativos: melhoria do bem-estar da população, no seu conjunto, e do seu estado social. Para Samagaio (1999:109) “enquanto o crescimento é material, por conseguinte quantitativo e objetivo, o desenvolvimento implica a obtenção de fins que transcendem o plano meramente económico, que servem a justiça, a cultura, a qualidade de vida, enfim, talvez, a felicidade".

Também para Diniz (2010) o desenvolvimento está para além do processo económico. Kulkarni e Rajam, referidos por Diniz (2010), incluem na definição de desenvolvimento o processo social e político através do qual uma sociedade tenta atingir uma série de objetivos, a saber: melhorar o nível de vida de todos os membros da sociedade nas diferentes vertentes de subsistência e emprego, saúde, educação e outros serviços sociais e culturais; contribuir para a criação de condições que conduzam ao amor-próprio; alargar o conjunto de oportunidades económicas e sociais à disposição dos membros da sociedade; assegurar que o processo de desenvolvimento é sustentável tanto do ponto de vista económico como ambiental.

Para Chris Gerry "perceber a natureza do processo de crescimento económico constitui um ponto de partida fundamental para elaborarmos estratégias de desenvolvimento mais eficazes” (Diniz, 2010:Prefácio). Para Gerry a questão essencial prende-se com a forma de combinar as diferentes variáveis que mais contribuem para o crescimento económico e bem-estar humano a fim de instalar um processo de desenvolvimento sustentado e sustentável.

A definição de desenvolvimento sustentável mais comummente referida na literatura tem origem no Relatório Brundtland, produzido em 1987 pela Comissão Mundial sobre o Ambiente e Desenvolvimento, que considera o desenvolvimento sustentável como o "desenvolvimento que dá resposta às necessidades do presente sem comprometer a capacidade das gerações”. Os aspetos fundamentais desta definição residem na importância do tempo e das perspetivas de longo prazo e no reconhecimento das potenciais relações de compromisso entre a resposta às necessidades do presente e a disponibilidade de recursos para dar resposta a necessidades do futuro.

O turismo na denominada economia verde referese às atividades que podem ser mantidas ou sustentadas indefinidamente nos seus contextos sociais, económicos e ambientais. O turismo sustentável é aquele que considera os impactos atuais e futuros nestas três esferas, tendo em conta as necessidades dos visitantes, da indústria, do ambiente e das comunidades hospedeiras. Não é uma forma especial de turismo mas todas as formas de turismo devem tentar ser mais sustentáveis (United Nations Environment Programme \& World Tourism Organization, 2012). De facto as linhas orientadoras para o desenvolvimento do turismo sustentável e as respetivas práticas de gestão são aplicáveis a todas as formas de turismo em todos os tipos de turismo, incluindo o turismo de massas e os vários nichos de segmentos turísticos. Também Ritchie e Crouch (2003) no seu livro sobre destinos competitivos numa perspetiva sustentável acrescentam que não é apenas o capital natural endógeno de cada destino que deve ser mantido mas todos os elementos de atração, os fatores de suporte e as infraestruturas devem também ser mantidas e geridas de forma sustentável se se pretender manter a competitividade económica e de mercado.

Em suma, estas orientações sobre os vários tipos de desenvolvimento mostram a urgência de apropriar políticas de desenvolvimento sustentáveis nos espaços rurais, para a preservação destes em termos sociais e ambientais, tendo estes espaços um papel fundamental na garantia da harmonia essencial para a evolução da humanidade. Os objetivos destas tipologias de desenvolvimento são pragmáticos: os instrumentos e as ações não só tratam de resolver problemas concretos, como dão maior importância à competitividade e à difusão das inovações e, do ponto de vista operativo, as iniciativas locais instrumentalizam-se através de formas flexíveis de organização, como as agências de desenvolvimento sustentadas numa rede de parcerias.

\section{QUESTÕES ESSENCIAIS NA PROMOÇÃO DO CRESCIMENTO/ DESENVOLVIMENTO DE UMA REGIÃO}

A questão que então se coloca é a de saber o que é que promove o crescimento/desenvolvimento de uma região. A relação entre especialização turística e crescimento económico é um dos principais tópicos de discussão nos estudos relacionados com a economia do turismo (Sinclair, 1998; Milne \& Ateljevic, 2001; Oh, 2005; Sequeira \& Nunes, 2008; Figini \& Vici, 2010; Matarrita-Cascante, 2010; Haugland et al., 2011). A revisão da literatura permite identificar cinco questões essenciais que parecem estar na base do desenvolvimento e prosperidade de uma região no que ao turismo diz respeito.

Atração de investimento externo para a região

Uma das primeiras questões que se coloca em relação ao desenvolvimento económico das regiões prende-se com a falta 
de suporte financeiro dos Governos face às regiões que faz com que estas tenham que conseguir sobreviver tirando o melhor partido possível da disponibilidade de investimento local e neste âmbito as regiões que querem desenvolver os seus destinos devem criar capacidade para atrair investimento externo para a área (Costa, 2006). O paradoxo do desenvolvimento global com base no local é defendido por Porter (1998) que argumenta que as vantagens competitivas da economia global assentam nas coisas locais - trata-se de conhecimento e relações que concorrentes distantes não conseguem imitar.

As regiões com recursos turísticos endógenos ricos podem especializar-se na produção e exportação de produtos que lhe tragam vantagens competitivas. Para além disso, deve evitar-se ao máximo recorrer às importações, as matériasprimas devem ser produzidas localmente, deve ser dada prioridade ao emprego de locais e, assim, este crescimento cumulativo irá espalhar-se para outras áreas do território com o desenvolvimento a ser induzido do centro para a periferia e não o contrário (Costa, 2006). De facto, as regiões precisam de se desenvolver de dentro para fora, isto é, precisam de cooperar localmente para poder competir num mundo global e globalizado (Milne \& Ateljevic, 2001; Coe et al., 2004).

$\mathrm{O}$ processo de desenvolvimento sustentável nas sociedades modernas está ligado à disponibilidade e ao acesso ao crédito para o crescimento dos negócios que promovem as atividades de produção de cada setor económico. A atividade turística é fundamental pela iniciativa privada e envolve um amplo leque de oportunidades para o desenvolvimento da oferta de produtos e serviços. Assim, é imprescindível a disponibilidade de créditos em quantidade e em condições adequadas às micro, medias e grandes empresas no setor do turismo.

Organização em rede e o estabelecimento de parcerias

A segunda questão é decorrente da anterior já que está intimamente ligada a esta cooperação local. Isto é, uma questão essencial no desenvolvimento das regiões é a organização em rede e o estabelecimento de parcerias entre os diferentes atores locais (Buhalis \& Cooper, 1998; Denicolai, Cioccarelli \& Zucchella, 2010a; Fyall, Garrod \& Wang, 2012; Sarmaniotis, Wickens \& Sigala, 2014). No turismo é premente a necessidade de informação de qualidade e em tempo real sobretudo quando estão envolvidos um vasto número de fornecedores do produto total. A este respeito a organização em rede no turismo sempre foi mais uma necessidade do que uma inovação (Buhalis \& Egger, 2008).

Para Costa e Buhalis (2006:202) “o produto turístico compreende um conjunto de ambientes físicos, sociais e culturais cujo sucesso depende da superestrutura montada para o gerir e vender. Para os autores esta superestrutura inclui as organizações inerentes às principais atividades económicas previstas na Conta Satélite do Turismo da Organização Mundial de Turismo (alojamento, alimentação e bebidas, transportes, operadores turísticos, agentes de viagens e guias turísticos, rent-a-car, serviços culturais e atividades de recreação e lazer) ao que é necessário acrescentar a infraestrutura, as instalações e a eficácia da governança (estratégia orientadora comum para todas as empresas e organizações) necessárias no destino.

Há vários contributos na literatura que permitem validar a importância da colaboração e organização em rede (Jamal \& Getz, 1995; Buhalis, Jafari \& Werthner, 1996; Raco, 1999; Tinsley \& Lynch, 2001; MacKinnon, Cumbers \& Chapman, 2002; Jamal \& Jamrozy, 2006; Manente \& Minghetti, 2006; Baggio, Corigliano \& Tallinucci, 2007; Scott, Cooper \& Baggio, 2008; Denicolai, Cioccarelli \& Zucchella, 2010b; Beritelli, 2011; Zach \& Racherla, 2011; Mistilis, Buhalis \& Gretzel, 2014). Jamal e Jamrozy (2006) definem uma estrutura para a gestão integrada de destinos baseada na sustentabilidade e em princípios de colaboração, adotando uma visão de sistema onde os espaços ecológicos, físicos e sociais estão ligados por vários tipos de redes e estruturas institucionais; para Scott et al. (2008) os destinos podem ser considerados redes de colaboração de organizações complementares na produção de um serviço e a coesão da rede interorganizacional no destino, medida pela análise de rede, é vista como um indicador de eficácia; Tinsley e Lynch (2001) discutem o estabelecimento de redes entre pequenas empresas turísticas e a sua contribuição para o desenvolvimento do destino; Baggio (2007) estuda a rede formada pelos websites de um destino turístico e conclui que a análise estatística das redes pode mostrar de forma fiável a estrutura de uma rede social peculiar, propondo coeficientes de clustering e de associação para medir quantitativamente até onde vai ai colaboração e a cooperação entre os intervenientes de um destino turístico.

$\mathrm{Na}$ atividade turística de um destino e na construção dos diferentes produtos desse mesmo destino é necessário envolver todos os intervenientes, sendo também vários os contributos na literatura sobre este aspeto concretamente a teoria dos stakeholders e a sua importância para a gestão estratégica de destinos (Sautter \& Leisen, 1999; Clement, 2005; Sheehan \& Ritchie, 2005; Spyriadis et al., 2009; Sigala \& Marinidis, 2010; Strobl \& Peters, 2013; Mistilis, Buhalis \& Gretzel, 2014). Os stakeholders (neste trabalho identificados como intervenientes) definem-se como sendo "qualquer entidade que é influenciada ou pode influenciar a execução das atividades da organização, ou seja, tem um interesse legítimo nas atividades da organização e que tem o poder de afetar o desempenho da mesma" (adaptado de Spyriadis et al. (2009:3)). Assim, o sucesso do destino depende da colaboração permanente entre os diferentes intervenientes envolvidos na cadeia de valor do turismo na prestação de experiências únicas e memoráveis ao turista (Beritelli, 2011; Zach \& Racherla, 2011). De acordo com os autores há um paradoxo interessante para as organizações envolvidas nesta cadeia de valor. Por um lado, elas têm que comercializar os seus próprios produtos e serviços e competir por um número limitado de turistas presentes no destino e, ao mesmo tempo, têm que cooperar e coordenar-se entre si concentrando-se no produto total a oferecer no destino. Esta é uma tarefa extremamente complexa já que os destinos têm inúmeros intervenientes que operam de acordo com as suas próprias ideias, objetivos e recursos. Para além disso, 
sendo o tecido empresarial turístico nos destinos constituído essencialmente por pequenas e médias empresas implicadas na construção de um produto comum (Buhalis \& Main, 1998; Sinclair, 1998; Koh \& Hatten, 2002; Withalm, Woelfel \& Smolak, 2007; Sigala, 2009), é premente a necessidade de estabelecer redes de negócios entre as empresas e estreitar a cooperação que por sua vez permite aos fornecedores turísticos melhorar o seu poder de negociação com operadores turísticos poderosos. Para Costa e Buhalis (2006:201) “ao nível regional e local os empresários expandem os seus negócios, associam-se entre si e criam economias locais e regionais que desafiam o predomínio das nações"

\section{A criação de produtos-território assentes em clusters para o turismo}

A terceira questão essencial ao desenvolvimento económico das regiões é a criação de produtos-território com oferta de experiências únicas e inesquecíveis o que deverá constituir o cerne da competitividade entre destinos. Para Costa (2006) o que é diferenciável e não comparável são os territórios e por isso deve ser colocada ênfase no desenvolvimento e promoção de territórios em vez de produtos. O marketing de lugares define, o produto como o suporte geográfico de atividades e simultaneamente um produto complexo, incluindo um conjunto de atividades, produtos, serviços e infraestruturas que servem as funções residencial, económica, social, cultural, turismo e lazer (Kastenholz, 2002).

Antes de se definir uma estratégia de desenvolvimento económico para uma região ou país (Smith, 1994), éindispensável reconhecer os seus principais recursos económicos, naturais, culturais e patrimoniais. No caso do turismo é necessário identificar os potenciais recursos geradores de turismo, com características distintivas e eventualmente únicas que permitam desenvolver produtos turísticos. Para Baptista (1990), produto turístico é uma mistura de tudo o quanto uma pessoa pode consumir, utilizar, experimentar, observar e apreciar durante uma viagem ou estada.

Porter foi o primeiro autor a utilizar o termo cluster para descrever um conjunto de empresas que formam um conglomerado para competir com outras, não pertencentes ao agrupamento, ou com outro cluster. Ainda segundo o autor, em função da proximidade geográfica, as empresas participantes do cluster se aproveitam de vários benefícios tais como especialização de fornecedores e recursos humanos, troca de conhecimento, competição e cooperação além do contínuo aprendizado pela interação cotidiana com fornecedores e clientes (Porter, 2000).

A existência de canais ativos é tão importante como a concentração e sem canais ativos mesmo uma massa crítica de empresas relacionadas não consubstancia uma produção local ou sistema social e, como tal, não opera como um cluster (Rosenfeld, 1997; Porter, 1998). Para os autores são as dinâmicas do cluster, e não o tamanho ou as capacidades individuais das empresas, que são a chave para a sinergia e como tal para a sua competitividade.

O conceito de rede, atrás referido, aparece na literatura, não raras vezes, intimamente relacionado com o conceito de cluster. Rosenfeld (1997), para quem o conceito de rede se traduz basicamente na cooperação entre empresas, defende que as redes são o resultado de clusters maturos e ativos. De facto, para o autor os clusters são sistemas onde a qualidade de membro se baseia simplesmente na interdependência e na contribuição para o funcionamento do sistema. O autor (op. Cit.) elenca algumas comparações entre redes e clusters que a seguir se apresentam.

Os clusters e as redes são vitais para o desenvolvimento regional já que aumentam a produtividade, o desempenho, a capacidade de inovação e a massa crítica dos negócios locais (Novelli, Schmitz \& Spencer, 2006). Para as autoras, ao nível regional e ao nível sectorial, é importante identificar quem está no mercado e examinar clusters potenciais que possam ser suportados pelas autoridades regionais e nacionais para o benefício das empresas locais.

São vários os contributos na literatura acerca da importância de criar clusters para o turismo com base nos produtos que o destino pode oferecer (Michael, 2003; Costa, 2006; Novelli, Schmitz \& Spencer, 2006). O objetivo de criar clusters e redes no turismo é destacar a disponibilidade de certas atividades num destino ou região e conseguir que as pequenas e médias empresas, que normalmente trabalham de forma isolada, cooperem e criem um produto turístico local (Novelli, Schmitz \& Spencer, 2006). As autoras dão como exemplo um projeto de desenvolvimento de um cluster associado ao turismo de saúde no sul de Inglaterra que permitiu às pequenas e médias empresas da região terem sucesso na integração de um cluster sustentado. O referido exemplo possibilita a identificação das diferentes áreas de atuação que permitiram implementar com sucesso um cluster turístico, a saber, a estimulação do trabalho em rede (através de workshops e reuniões); o fornecimento de infraestruturas (a partilha de infraestruturas de formação e reunião como por exemplo o campus da universidade e as instalações das empresas), a transferência de conhecimento (trabalho em colaboração promovido por especialistas em diversas áreas) e a partilha de melhores práticas (benchmarking de práticas especificas de referência).

Michael (2003) destaca a importância da estrutura e da escala dos clusters quando aplicados ao contexto do turismo. Destaca sobretudo a criação de oportunidades económicas e sociais em pequenas comunidades através do desenvolvimento de clusters de empresas complementares que podem coletivamente fornecer um conjunto de atributos que constituam um produto regional especializado. De facto, o desempenho das economias regionais é fortemente influenciado pelo poder dos clusters locais e pela vitalidade e pluralidade da inovação (Costa \& Buhalis, 2006). Por sua vez, a identificação dos produtos que podem ter vantagem comparativa terá que assentar, por um lado, na análise compreensiva dos recursos do destino e, por outro lado, na análise do mundo competitivo em que o destino se insere.

Importância da governança

Em quarto lugar surge a importância da governança das redes, mas também a importância da governança no 
planeamento local. Em relação a esta, para Costa (2006), o desenvolvimento deve ser estimulado ao nível regional o que compreende, por um lado, a gestão dos recursos endógenos (planeamento físico) e, por outro lado, do ponto de vista da organização e coordenação do território inclui a superestrutura necessária para o planeamento e gestão (planeamento estratégico). Neste sentido, o turismo, enquanto atividade que ocorre em um determinado espaço, com características socioculturais e económicas singulares, determina um tipo de ocupação e de impactos que necessitam ser administrados de forma eficiente, sob o risco de ser altamente danoso ao ambiente em que se desenvolve.

A Organização Mundial de Turismo (2010) discute a questão da governança dos destinos turísticos como sendo o processo através do qual cada organização de gestão de destinos atua e desempenha as suas funções. Define as atividades operacionais, as expectativas, as prioridades e os objetivos de uma organização. A governança do destino diz respeito ao desenvolvimento e implementação de uma política coesa, de um sistema de gestão consistente e à existência de uma estrutura de gestão eficiente no seio de uma organização de gestão de destinos (Pechlaner, Volgger \& Herntrei, 2012).

No que diz respeito à importância da governança das redes, se o sucesso do destino depende da colaboração permanente entre os diversos intervenientes, então, esta colaboração implica que se devem obter elevados níveis de coordenação que por sua vez só podem ser obtidos se houver uma estrutura de governança instituída. Esta é implementada através das estruturas organizacionais exigidas pelos clusters turísticos orientados para o produto (atrás referidos) que devem ter a capacidade de gerir os interesses fragmentados envolvidos na atividade turística e assegurar que a sua localização no território é feita para que as organizações não ponham em perigo o próprio território (Costa \& Buhalis, 2006). Para os autores, esta governança não deve deixar de lado o formalismo administrativo embora também tenha que se ter em conta as características do destino e do mercado a conquistar; do ponto de vista legislativo, a governança implica uma estrutura abrangente que permita aos atores turísticos a flexibilidade suficiente para tomar decisões e para definir e promover produtos.

A importância da governança das redes tem sido largamente defendida e referida na literatura através do conceito de distritos industriais (industrial districts) (Raco, 1999; Hjalager, 2000; MacKinnon, Cumbers \& Chapman, 2002; Coe et al., 2004; Mottiar \& Ryan, 2006; Sainaghi, 2006; Scott, Cooper \& Baggio, 2008) como sendo áreas onde se tem verificado uma significativa prosperidade apesar da imensa competitividade ao nível de regiões. De facto, na literatura, as regiões têm surgido como conceito central na teoria do desenvolvimento económico (Candela \& Figini, 2009) e as ligações institucionais surgem como base da competitividade e coesão das mesmas (Raco, 1999; MacKinnon, Cumbers \& Chapman, 2002; Kong, 2007; Rigall-I-Torrent, 2008; Zemła, 2014). Para Diniz (2010) as instituições determinam a prontidão com que a sociedade obtém vantagens das diversas oportunidades para aumentar o seu nível de bem-estar. "Os modelos de crescimento/desenvolvimento indicam quais as variáveis críticas ao processo de crescimento mas são as instituições os incentivos que determinam se o crescimento/ desenvolvimento ocorrerá ou não" (Diniz, 2010:22). Assim, só se as instituições forem compatíveis com a poupança, o investimento, a intermediação financeira, o comércio e o investimento internacional, o investimento em capital humano, a inovação/investigação e um vasto conjunto de atividades criativas e empresariais é que o crescimento/desenvolvimento pode ocorrer. As instituições têm uma relação íntima com o governo, o estado e a política e esta pode deteriorar-se ou melhorar conforme se distorcem ou não os incentivos do seu papel positivo na promoção do crescimento da atividade humana. Ainda para o mesmo autor (op. Cit.), o caminho para o crescimento/desenvolvimento económico é claro e passa por escolhas políticas que intensifiquem o papel das instituições para que permitam: o aumento da liberdade económica e pessoal; a proteção dos direitos de propriedade; a eliminação das barreiras à mudança económica; e a promoção de incentivos à inovação evitando as atividades destrutivas e protecionistas. Volgger \& Pechlaner (2014) estudam a relação existente entre a capacidade de trabalhar em rede e o sucesso do destino e das organizações de gestão de destinos. Concluem que o efeito da capacidade de trabalhar em rede surge através do aumento da autoridade reconhecida a estas organizações, isto é, surge com a promoção da aceitação e o aumento do poder das organizações de gestão de destinos no seio da rede, no destino.

Importância dos critérios económicos

Uma última questão que está na base do desenvolvimento e prosperidade de uma região no que ao turismo diz respeito é a importância dos critérios económicos para as empresas mas sem perder de vista o respeito pelo território e a sustentabilidade. Só os produtos economicamente viáveis é que são sustentáveis economicamente e só esses é que interessam às empresas. É a consciência que o sucesso individual de cada negócio incorpora a noção de realização económica comum mas também o respeito pelo território que o suporta, que cria a ligação entre as redes e o território e fornece a orientação para aquilo que deve ser o turismo sustentável (Costa \& Buhalis, 2006). Ainda, se o processo de crescimento/desenvolvimento económico levar à escassez dos recursos que a terra tem para fornecer, então essa deverá constituir uma preocupação a resolver. Sendo que a ciência económica trata do dilema existente entre recursos escassos com usos alternativos a afetar na produção de bens e serviços, então, esta mesma ciência deve ser posta ao serviço do desenvolvimento sustentável. Este termo refere-se à taxa de crescimento que o mundo é capaz de suster indefinidamente e tecnicamente refere-se à taxa de crescimento económico que pode ser mantida sem diminuir o stock efetivo de recursos (Diniz, 2010).

Os princípios do turismo sustentável podem traduzir-se em práticas de gestão aplicáveis a todo tipo de empresa, em qualquer destino turístico. Estes princípios têm como propósito 
minimizar os impactos negativos e maximizar os benefícios da atividade turística no entorno sociocultural, ambiental e empresarial.

\section{Conclusão}

A revisão da literatura inerente ao presente estudo pretende demonstrar o contributo do turismo para o desenvolvimento das regiões, devido à utilização, exploração e usufruto dos recursos, nomeadamente endógenos, à criação e melhoria das atividades e dos produtos característicos do turismo próprios de cada região. O turismo leva, ainda, à expansão económica local, com a criação de emprego e serviços, preferencialmente adequados e compatíveis com o produto regional em foco. Como ganho adicional reforça o sentido da preservação sustentada do património natural (nomeadamente o paisagístico), cultural, histórico e tradicional dos territórios, não só importantes por serem o objeto de exploração económica, mas também por contribuir para a sustentabilidade económica de uma herança comum a manter e a usufruir. As entidades locais e regionais, públicas, privadas e associativas do turismo, assumem um papel decisivo no desenvolvimento regional, não só no planeamento e execução dos objetivos negociados e delineados conjuntamente, como também no papel de fomento, por atraírem investimento externo, contribuindo para o impulso do desenvolvimento turístico e para o bem-estar das populações locais.

\section{REFERÊNCIAS}

Baggio, R. 2007. The web graph of a tourism system. PHYSICA A, 379: 727-734.

Baggio, R., Corigliano, M. A., \& Tallinucci, V. 2007. The Websites of a Tourism Destination: A Network Analysis In M. Hitz, M. Sigala, \& J. Murphy (Eds.), Information and Communication Technologies in Tourism 2007, Vol. 6: 279288: Springer Vienna.

Baptista, M. 1990. OTurismo na Economia - uma abordagem técnica, economia, social e cultural. Lisboa: Instituto Nacional de Formação Turística.

Beritelli, P. 2011. Cooperation Among Prominent Actors In A Tourist Destination. Annals of Tourism Research, 38(2): 607629.

Buhalis, D., \& Cooper, C. 1998. Competition or Co-operation? Small and medium sized tourism enterprises at the destination. In E. Laws, H. W. Faulkner, \& G. Moscardo (Eds.), Embracing and managing change in tourism: international case studies. London: Routledge.

Buhalis, D., \& Egger, R. 2008. eTourism Case Studies management and marketing issues. Oxford: Elsevier Ltd.

Buhalis, D., Jafari, J., \& Werthner, H. 1996. Information Technology and the Reengineer of Tourism. Paper presented at the ENTER 96 - The International Conference on
Information and Telecommunication Technologies in the Field of Tourism, Innsbruck, Austria.

Buhalis, D., \& Main, H. 1998. Information technology in peripheral small and medium hospitality enterprises: strategic analysis and critical factors. International Journal of Contemporary Hospitality Management, 10(5): 198-202.

Candela, G., \& Figini, P. 2009. Destination unknown. Is there any economics beyond tourism areas?, Review of Economic Analysis, Forthcoming.

Clement, R. W. 2005. The lessons from stakeholder theory for U.S. business leaders. Business Horizons 48: 255-264.

Coe, N. M., Hess, M., Yeung, H. W.-c., Dicken, P., \& Henderson, J. 2004. 'Globalizing' regional development: a global production networks perspective. Transactions of the Institute of British Geographers, 29(4): 468-484.

Costa, C. 2006. Tourism Planning, Development and the Territory. In D. Buhalis, \& C. Costa (Eds.), Tourism Management Dynamics - trends, management and tools: 236-243: Elsevier, Ltd.

Costa, C., \& Buhalis, D. 2006. Synergies between Territorial Planning and Strategic Management: a prospective analysis. In CEG (Ed.), Desenvolvimento e Território: Espaços Rurais Pós-Agrícolas e Novos Lugares de Turismo e Lazer. Lisboa: Centro de Estudos Geográficos, Universidade de Lisboa.

Denicolai, S., Cioccarelli, G., \& Zucchella,A. 2010a. Resourcebased local development and networked core-competencies for tourism excellence. Tourism Management, 31(2): 260-266.

Denicolai, S., Cioccarelli, G., \& Zucchella,A. 2010b. Resourcebased local development and networked core-competencies for tourism excellence. Tourism Management, 31: 206-266.

Diniz, F. 2010. Crescimento e Desenvolvimento Económico - Modelos e Agentes do Processo (2 ${ }^{\mathrm{a}}$ ed.). Lisboa: Edições Sílabo.

Figini, P., \& Vici, L. 2010. Tourism and growth in a cross section of countries. Tourism Economics, 16(4): 789-805.

Fyall, A., Garrod, B., \& Wang, Y. 2012. Destination collaboration: A critical review of theoretical approaches to a multi-dimensional phenomenon. Journal of Destination Marketing \& Management, 1(1-2): 10-26.

Haugland, S. A., Ness, H., Grønseth, B.-O., \& Aarstad, J. 2011. Development of tourism destinations: An Integrated Multilevel Perspective. Annals of Tourism Research, 38(1): 268-290.

Hjalager, A.-M. 2000. Tourism destinations and the concept of industrial districts. Tourism \& Hospitality Research, 3: 199213.

Jamal, T., \& Jamrozy, U. 2006. Collaborative Networks and Partnerships for Integrated Destination Management. In D. Buhalis, \& C. Costa (Eds.), Tourism Management Dynamics - Trends, management and tools. Oxford: Elsevier Butterworth-Heinemann.

Jamal, T. B., \& Getz, D. 1995. COLLABORATION THEORY 
AND COMMUNITY TOURISM PLANNING. Annals of Tourism Research, 22(1): 186-204.

Koh, K. Y., \& Hatten, T. S. 2002. The Tourism Entrepreneur: The Overlooked Player in Tourism Development Studies. International Journal of Hospitality \& Tourism Administration, 3(1): 21-48.

Kong, T. 2007. A Selective Review of Recent Developments in the Economic Growth Literature. Asian-Pacific Economic Literature, 21(1): 1-33.

Kuznets, S. 1973. Modern Economic Growth: Findings and Reflections. The American Economic Review, 63(3): 247258.

MacKinnon, D., Cumbers, A., \& Chapman, K. 2002. Learning, innovation and regional development: a critical appraisal of recent debates. Progress in Human Geography, 26(3): 293 311.

Manente, M., \& Minghetti, V. 2006. Destination Management Organizations and Actors. In D. Buhalis, \& C. Costa (Eds.), Tourism Management Dynamics - consumers, products and industry: Elsevier. Ltd.

Matarrita-Cascante, D. 2010. Beyond Growth Reaching Tourism-Led Development. Annals of Tourism Research, 37(4): 1141-1163.

Michael, E. J. 2003. Tourism micro-clusters. Tourism Economics, 9(2): 133-145.

Milne, S., \& Ateljevic, I. 2001 . Tourism, economic development and the global-local nexus: theory embracing complexity. Tourism Geographies, 3(4): 369-393.

Mistilis, N., Buhalis, D., \& Gretzel, U. 2014. Future eDestination Marketing: Perspective of an Australian Tourism Stakeholder Network. Journal of Travel Research: 1-13.

Mottiar, Z., \& Ryan, T. 2006. The Role of SMEs in Tourism Development: an Industrial District Approach Applied to Killarney, Ireland. In R. Thomas, \& M. Augustyn (Eds.), Tourism in the New Europe: perspectives on SME policies and practices: Elsevier.

Novelli, M., Schmitz, B., \& Spencer, T. 2006. Networks, clusters and innovation in tourism: a UK experience. Tourism Management, 27: 1141-1152.

Oh, C.-O. 2005. The contribution of tourism development to economic growth in the Korean economy. Tourism Management, 26: 39-44.

Pechlaner, H., Volgger, M., \& Herntrei, M. 2012. Destination management organizations as interface between destination governance and corporate governance. Anatolia: An International Journal of Tourism and Hospitality Research, 23(2): 151-168.

Porter, M. 2000. Location, competition, and economic development: Local clusters in a global economy. Economic development quarterly, 14(1): 15-34.

Porter, M. E. 1998. Clusters and the New Economics of
Competition. Harvard Business Review, Nov-Dec: 77-90.

Raco, M. 1999. Competition, Collaboration and the New Industrial Districts: Examining the Institutional Turn in Local Economical Development. Urban Studies, 36(5-6): 951-968.

Rigall-I-Torrent, R. 2008. Sustainable development in tourism municipalities: The role of public goods. Tourism Management, 29: 883-897.

Ritchie, J. R. B., \& Crouch, G. I. 2003. The competitive destination: a sustainable tourism perspective: $\mathrm{CABI}$ Publishing.

Rosenfeld, S. A. 1997. Bringing business clusters into the mainstream of economic development. European Planning Studies, 5(1): 3-23.

Sainaghi, R. 2006. From contents to processes: Versus a dynamic destination management model. Tourism Management, 27: 1053-1063.

Samagaio, F. 1999. Desenvolvimento: uma noção entre o imaginário e a realidade. Sociologia - Revista da Faculdade de Letras da Universidade do Porto, IX(1): 103 - 142.

Sarmaniotis, C., Wickens, E., \& Sigala, M. 2014. Evaluating the performance of destination marketing systems (DMS): stakeholder perspective. Marketing Intelligence \& Planning, 32(2): 208-231.

Sautter, E. T., \& Leisen, B. 1999. Managing Stakeholders A Tourism Planning Model. Annals of Tourism Research 26(2): 312-328.

Scott, N., Cooper, C., \& Baggio, R. 2008. Destination Networks Four Australian Cases. Annals of Tourism Research, 35(1): 169-188.

Sequeira, T. N., \& Nunes, P. M. 2008. Does tourism influence economic growth? A dynamic panel data approach. Applied Economics, 40(18): 2431-2441.

Sheehan, L. R., \& Ritchie, J. R. B. 2005. Destination Stakeholders Exploring Identity and Salience Annals of Tourism Research, 32(3): 711-734.

Sigala, M. 2009. Destination Management Systems (DMS): A Reality Check in the Greek Tourism Industry. In W. Höpken, U. Gretzel, \& R. Law (Eds.), Information and Communication Technologies in Tourism 2009: Springer Vienna.

Sigala, M., \& Marinidis, D. 2010. DMOs, e-Democracy and Collaborative Destination Management: An Implementation Framework. In U. Gretzel, R. Law, \& M. Fuchs (Eds.), Information and Communication Technologies in Tourism 2010 Vol. 7: 235-246. Wien: Springer-Verlag.

Sinclair, M. T. 1998. Tourism and economic development: A survey. Journal of Development Studies, 34(5): 1-51.

Smith, S. 1994. The Tourism product. Annals of Tourism Research, 21(3): 582-595.

Spyriadis, T., Fletcher, J., Fyall, A., \& Carter, R. 2009. Evaluating Performance of Organisations Operating in 
Composite Industrial Environments: the Case of Tourism Destination Management Organisations: [online], disponível: http: / / www.pma.otago.ac.nz./pma-cd/papers/1092.pdf, 14/10/2009.

Strobl, A., \& Peters, M. 2013. Entrepreneurial reputation in destination networks. Annals of Tourism Research, 40: 5982.

Tinsley, R., \& Lynch, P. 2001. Small tourism business networks and destination development. Hospitality Management, 20: 367-378.

United Nations Environment Programme, \& World Tourism Organization. 2012. Tourism in the Green Economy Background Report

Volgger, M., \& Pechlaner, H. 2014. Requirements for destination management organizations in destination governance:
Understanding DMO success. Tourism Management, 41(0): 64-75.

Withalm, J., Woelfel, W., \& Smolak, I. 2007. Collaboration Agents. In M. Sigala, L. Mich, \& J. Murphy (Eds.), Information and Communication Technologies in Tourism 2007, Vol. 7: 289-300. Viena: Springer.

World Tourism Organization. 2010. Survey on Destination Governance. Madrid UNWTO.

Zach, F., \& Racherla, P. 2011. Assessing the Value of Collaborations in Tourism Networks: A Case Study of Elkhart County, Indiana Journal of Travel \& Tourism Marketing, 28: 97-110.

Zemła, M. 2014. Inter-destination cooperation: Forms, facilitators and inhibitors - The case of Poland. Journal of Destination Marketing \& Management(0). 